\title{
具有双孔径分布的介孔 $\mathrm{C}-\mathrm{Al}_{2} \mathrm{O}_{3}-\mathrm{TiO}_{2}$ 纳米复合材料的合成及 其红外发射率
}

\author{
李国显 王 涛 何建平 ${ }^{*}$ 周建华 薛海荣 马一鸥 胡园园 \\ (南京航空航天大学材料科学与技术学院, 南京 210016)
}

摘要: 以嵌段共聚物 $\mathrm{F} 127\left(\mathrm{PEO}_{106} \mathrm{PPO}_{70} \mathrm{PEO}_{106}, M_{\mathrm{w}}=12600\right)$ 为模板剂, 异丙醇铝和钛酸四丁酯为金属源, 低 分子量的酚醋树脂为碳源, 通过溶胶-凝胶三元共组装法合成了具有双孔径分布的 $\mathrm{C}^{-} \mathrm{Al}_{2} \mathrm{O}_{3}-\mathrm{TiO}_{2}$ 纳米复合材料. 用 X射线衍射(XRD)、透射电子显微镜(TEM)、扫描电子显微镜(SEM)及 $\mathrm{N}_{2}$ 吸附-脱附对该复合材料进行结构表 征. 结果显示, 当铝钛原子的摩尔比为 1:10 时, 对应的纳米复合材料具有较好的有序介孔结构, 其双孔径分别 为 3.9 和 $6.5 \mathrm{~nm}$, 比表面积可达 $259 \mathrm{~m}^{2} \cdot \mathrm{g}^{-1}$, 孔容 $0.37 \mathrm{~cm}^{3} \cdot \mathrm{g}^{-1}$. 以三元乙丙橡胶(EPDM)为粘结剂, 与介孔纳米 复合材料混合制备涂层. 通过调节复合材料中铝钠摩尔比和涂层厚度, 红外发射率在 0.450-0.617之间可调.

关键词: 介孔; 有序; $\mathrm{C}-\mathrm{Al}_{2} \mathrm{O}_{3}-\mathrm{TiO}_{2}$ 纳米复合材料; 双孔径; 红外发射率 中图分类号: O648; TB33

\section{Synthesis and Infrared Emissivity of Ordered Mesoporous $\mathrm{C}-\mathrm{Al}_{2} \mathrm{O}_{3}-\mathrm{TiO}_{2}$ Nanocomposites with a Dual Pore System}

\author{
LI Guo-Xian WANG Tao HE Jian-Ping* ZHOU Jian-Hua XUE Hai-Rong \\ MA Yi-Ou HU Yuan-Yuan
}

(College of Material Science and Technology, Nanjing University of Aeronautics and Astronautics, Nanjing 210016, P. R. China)

\begin{abstract}
C}_{-} \mathrm{Al}_{2} \mathrm{O}_{3}-\mathrm{TiO}_{2}$ nanocomposites with a dual pore system were synthesized via the sol-gel tri-constituent co-assembly process using an amphiphilic triblock copolymer $\mathrm{F} 127\left(\mathrm{PEO}_{106} \mathrm{PPO}_{70} \mathrm{PEO}_{106}\right.$, $M_{\mathrm{w}}=12600$ ) as a template, aluminum isopropoxide and tetrabutyl titanate as metallic sources and resol as an organic precursor. X-ray diffraction (XRD), transmission electron microscopy (TEM), scanning electron microscopy (SEM), and nitrogen adsorption/desorption were used to characterize the structures of these nanocomposites. The results show that the nanocomposite with a Al/Ti molar ratio of 1:10 has ordered mesoporous structures with a dual system of 3.9 and $6.5 \mathrm{~nm}$, a high specific surface area of $259 \mathrm{~m}^{2} \cdot \mathrm{g}^{-1}$ and a pore volume of $0.37 \mathrm{~cm}^{3} \cdot \mathrm{g}^{-1}$. Low infrared emissivity coatings were obtained using the ethylenepropylene-diene monomer (EPDM) as an adhesive and the ordered mesoporous $\mathrm{C}-\mathrm{Al}_{2} \mathrm{O}_{3}-\mathrm{TiO}_{2}$ nanocomposites as a filling. As the $\mathrm{Al} / \mathrm{Ti}$ molar ratio and coat thickness changed the infrared emissivities changed from 0.450 to 0.617 .
\end{abstract}

Key Words: Mesoporous; Ordered; $\quad \mathrm{C}_{-} \mathrm{Al}_{2} \mathrm{O}_{3}-\mathrm{TiO}_{2}$ nanocomposites; Dual-porosity; Infrared emissivity

Received: July 13, 2010; Revised: October 9, 2010; Published on Web: December 13, 2010.

"Corresponding author. Email: jianph@nuaa.edu.cn; Tel: +86-25-52112626.

The project was supported by the Aeronautical Science Foundation of China (2007ZF52061) and National Natural Science Foundation of China (50871053).

航空科学基金(2007ZF52061)和国家自然科学基金(50871053)资助项目

C Editorial office of Acta Physico-Chimica Sinica 
有序介孔碳是近年来迅速兴起的一类新型功能 材料, 具有规则的孔道结构, 孔径分布窄, 孔径尺寸 可以在大范围内连续调节, 且具有较大的比表面积 和孔容, 较好的热与化学稳定性, 且易于形成多功能 的碳基复合材料, 在催化、吸附、分离及光、电、磁等 许多领域有着潜在的应用前景 ${ }^{[1-6]}$.

有序介孔碳基纳米复合材料已有应用于雷达隐 身的报道 ${ }^{[7]}$. 有序介孔碳基复合材料具有的多孔结 构显著地增大了材料的表面积, 微波进入时能够增 加多次反射的机会, 降低材料的反射率, 提高材料的 微波吸收能力, 同时质轻的碳材料还有利于减轻隐 身涂层的质量 ${ }^{[8-9]}$. 另外, 介孔材料的多孔结构可以 有效降低材料的复介电常数, 从而使材料与自由空 间更好地匹配, 使电磁波更多地进入材料内部而耗 散掉 ${ }^{[10-11]}$. 随着探测技术的发展, 多波段隐身成为军 事研究的重点, 其中最主要的就是兼容雷达隐身和 红外隐身. 介孔材料的多孔结构降低微波反射率的 同时, 增大了材料的红外发射率 ${ }^{[12]}$. 使用半导体金属 氧化物抑制目标体的红外发射率是目前常用的红外 隐身的技术路线, 如王自荣等 ${ }^{[13]}$ 将掺锡氧化铟粉末 经研磨后烧结, 与不同粘合剂粘合制成涂料, 研究其 发射率. 随 $\mathrm{SnO}_{2}$ 掺杂含量的增加, 其发射率先减小, 后增加, 当其摩尔分数为 $5 \%$ 左右时, 涂层的发射率 最低. 如果能够将半导体金属氧化物嵌入到有序纳 米孔道中, 实现碳基与金属氧化物在介孔尺度上的 有序复合, 则可以降低介孔材料的红外发射率, 实现 兼容隐身的目标. 通过硬模板法或软模板法能够实 现介孔碳与其他组分的复合. 如 Liu 等 ${ }^{[8]}$ 利用 SBA15 为硬模板制备了有序介孔碳与氧化硅的复合材 料,实验发现该复合材料的微波吸收能力随着介孔 碳含量的增加而显著增强. Zhao 等 ${ }^{[14-15]}$ 以有机-无机 三元共组装法合成了有序介孔碳-氧化物纳米复合 材料, 通过调节碳前驱体和氧化物前驱体的比例控 制复合材料中碳和氧化物的相对含量, 氧化物含量 可以从 0 变化到 $100 \%$.

为了拓宽介孔材料在军事隐身领域的应用, 使 其具有多功能隐身特性吸引了我们的关注. 我们前 期报道了采用三组分共组装直接制备有序介孔 C$\mathrm{Al}_{2} \mathrm{O}_{3}$ 复合材料的方法, 碳作为 “粘结剂”起到稳定有 序介孔结构的作用, 当氧化铝含量占 $50 \%(w)$ 时, 其 红外发射率为 $0.496^{[16]}$. 半导体材料是具有很好发展 前景的一类红外低发射率材料 ${ }^{[17-19]}$, 且在雷达波段 具有较高的吸收率 ${ }^{[20-21]}$. 因此, 将介孔碳材料和半导
体材料结合有可能获得具有多功能隐身性能的新型 材料. $\mathrm{TiO}_{2}$ 的价带由氧的 $2 p$ 带构成, 导带主要是钛 的 $3 d$ 带, 禁带宽度宽 (约为 $3 \mathrm{eV}$ ), 对可见光几乎不吸 收, 对近红外的反射比大, 发射率低 ${ }^{[22]}$. 为了引入功 能优异的半导体材料二氧化钛, 并进一步研究这种 碳-金属氧化物合成方法的适用性, 尝试将模板剂、 碳源和金属源混合共组装来制备介孔碳-金属氧化 物纳米复合材料. 本文选用低分子量的酚醛树脂、钛 酸四丁酯、异丙醇铝为前驱体, 三嵌段共聚物 F127 为结构导向剂合成介孔 $\mathrm{C}-\mathrm{Al}_{2} \mathrm{O}_{3}-\mathrm{TiO}_{2}$ 复合材料. 采 用 XRD、TEM、SEM 和氮气吸脱/脱附表征了材料的 结构特征, 通过测试该复合材料在 8-14 $\mu \mathrm{m}$ 波段的 红外发射率, 探讨了金属氧化物相对含量的变化对 红外发射率的影响.

\section{1 实验部分}

\section{1 试 剂}

$\mathrm{F} 127\left(M_{\mathrm{w}}=12600, \mathrm{PEO}_{106} \mathrm{PPO}_{70} \mathrm{PEO}_{106}\right.$, 美国 Sigma Aldrich 公司); 苯酚、甲醛及二甲苯(上海久亿化学试 剂有限公司), 无水乙醇(南京化学试剂有限公司), 盐 酸(上海化学试剂公司), 均为分析纯; 钛酸四丁酯 $\mathrm{Ti}\left(\mathrm{OC}_{4} \mathrm{H}_{9}\right)_{4}$ (国药集团化学试剂公司)和异丙醇铝 $\mathrm{Al}\left(\mathrm{OC}_{3} \mathrm{H}_{7}\right)_{3}$ (国药集团化学试剂公司)为化学纯; 三元 乙丙橡胶为江苏省江阴福为贸易有限公司提供; 去 离子水为自制.

\section{2 有序介孔 $\mathrm{C}-\mathrm{Al}_{2} \mathrm{O}_{3}-\mathrm{TiO}_{2}$ 纳米复合材料的制备}

通过酚醛树脂前驱体、钛酸四丁酯、异丙醇铝前 驱体和三嵌段共聚物 F127多组分共组装制备介孔 $\mathrm{C}-\mathrm{Al}_{2} \mathrm{O}_{3}-\mathrm{TiO}_{2}$ 纳米复合材料. 典型的制备过程是将 $2.0 \mathrm{~g}$ 模板剂 F127 溶于 $12.0 \mathrm{~mL}$ 无水乙醇中, 搅拌形 成透明的溶液 $\mathrm{A}$; 将 $2 \mathrm{~mL}$ 浓盐酸加入到 $6 \mathrm{~mL}$ 无水 乙醇中, 再缓慢加入 $3.40 \mathrm{~g}$ 钛酸四丁酯 $(0.01 \mathrm{~mol})$ 和 $0.204 \mathrm{~g}$ 异丙醇铝 $(0.001 \mathrm{~mol})$ 得到溶液 B. 缓慢滴加 $2.5 \mathrm{~g}$ 酚醛树脂的乙醇溶液 (resol, 质量分数为 $20 \%$, 按文献[23]中的方法制备)于溶液 A 中, 摚拌 $30 \mathrm{~min}$; 然后将溶液 $\mathrm{B}$ 缓慢加于溶液 $\mathrm{A}$ 中, 摚拌 $2 \mathrm{~h}$ 使其混合 均匀. 将混合物转移到平底蒸发血中, 在室温 $\left(25^{\circ} \mathrm{C}\right)$ 下溶剂蒸发 $24 \mathrm{~h}$, 放入干燥箱中 $70{ }^{\circ} \mathrm{C}$ 真空热聚合 $24 \mathrm{~h}$, 形成薄膜. 将其刮下放入瓷舟, 置于管式炉中, 在氮气氛围下, $350{ }^{\circ} \mathrm{C}$ 炦烧 $5 \mathrm{~h}$, 然后在 $500^{\circ} \mathrm{C}$ 陆烧 $2 \mathrm{~h}$ 得到介孔 $\mathrm{C}-\mathrm{Al}_{2} \mathrm{O}_{3}-\mathrm{TiO}_{2}$ 纳米复合材料, 焙烧过程 中升温速率为 $1^{\circ} \mathrm{C} \cdot \mathrm{min}^{-1}$. 通过调节异丙醇铝/钛酸 四丁酯的原料摩尔比可以得到不同组成的纳米复合 
材料(表 1), 介孔 $\mathrm{C}-\mathrm{Al}_{2} \mathrm{O}_{3}-\mathrm{TiO}_{2}$ 纳米复合材料标记为 $\operatorname{MCAT} x, x$ 代表样品中铝原子和钛原子的理论摩尔 比, 如 MCAT10 代表样品中铝原子和钛原子的理论 摩尔比为 10:1. 作为对比, $\mathrm{C}-\mathrm{Al}_{2} \mathrm{O}_{3}$ 和 $\mathrm{C}-\mathrm{TiO}_{2}$ 纳米复 合材料也按同样的方法制备, 钛酸四丁酯和异丙醇 铝的添加量均为 $10 \mathrm{mmol}$, 分别标记为 MCA 和 MCT. 有序介孔碳按同样的方法制备, 记为 OMC.

\section{3 涂层的制备}

首先进行铝基板预处理: 砂纸打磨 $\rightarrow$ 水洗 $\rightarrow$ 化 学除油 $\rightarrow$ 化学出光 $\rightarrow$ 水洗 $\rightarrow$ 烘干; 然后选用三元乙 丙橡胶为粘合剂溶于二甲苯中, 加入适量的粉末样 品, 混合球磨, 制得涂料; 最后使用涂覆棒将涂料涂 于铝基板上，自然烘干. 采用德国 ElektroPhysik MiniTest 600 测厚仪测试了涂层的厚度.

\section{4 材料的结构表征和性能测试}

采用德国 Bruker D8 ADVANCE X射线衍射仪 测定样品的 X射线衍射峰. 阴极用 $\mathrm{Cu}$ 靶 $K_{\alpha}$ 线 $(\lambda=$ $0.15406 \mathrm{~nm}), \mathrm{Ni}$ 滤光片, $\mathrm{X}$ 射线的管电压为 $40 \mathrm{kV}$, 管电流为 $40 \mathrm{~mA}$. 采用 Micrometric 2010 物理吸附仪 进行材料的物理吸附性能表征. TEM 图使用荷兰 FEI Tecnai $G^{2}$ 透射电子显微镜获得, 其加速电压为 $200 \mathrm{kV}$. 采用 Quanta 400FEG 型扫描电子显微镜观 察材料的表面状态并测试材料的能谱以分析材料的 微区构成. 采用中国科学院上海物理技术研究所研 制的 IR-2 型双波段发射率测量仪测量样品在室温 下, 8-14 $\mu \mathrm{m}$ 波段的平均发射率.

\section{2 结果与讨论}

\section{1 介孔 $\mathrm{C}-\mathrm{Al}_{2} \mathrm{O}_{3}-\mathrm{TiO}_{2}$ 纳米复合材料的结构性能}

利用低分子量的可溶性酚醛树脂、钛酸四丁酯、 异丙醇铝和F127多组分的共组装得到F127-高分 子-氧化铝-氧化钛纳米复合材料. 复合材料中, 酚醛 树脂与三嵌段共聚物 PEO-PPO-PEO 热稳定性差异 较大 ${ }^{[22]}$, 而氧化铝和氧化钛属于无机氧化物, 具有很

表 1 介孔 $\mathrm{C}-\mathrm{Al}_{2} \mathrm{O}_{3}-\mathrm{TiO}_{2}$ 纳米复合材料的制备条件

Table 1 Synthesis condition of mesoporous $\mathrm{C}_{-}-\mathrm{Al}_{2} \mathrm{O}_{3}-\mathrm{TiO}_{2}$ nanocomposites

\begin{tabular}{|c|c|c|c|c|c|c|}
\hline Sample & $\frac{n_{\mathrm{Ti}\left(\mathrm{OC}_{4} \mathrm{H}_{9}\right)_{4}}}{\mathrm{mmol}}$ & $\frac{n_{\mathrm{Al}\left(\mathrm{OC}_{3} \mathrm{H}_{7}\right)_{3}}}{\mathrm{mmol}}$ & $\frac{m_{\text {Resol }}}{\mathrm{g}}$ & $\frac{m_{\mathrm{F} 127}}{\mathrm{~g}}$ & $\frac{V_{\mathrm{HCl}}}{\mathrm{mL}}$ & $\frac{m_{\text {ethanol }}}{\mathrm{g}}$ \\
\hline MCAT0.1 & 10 & 1 & 2.5 & 2 & 2 & 18 \\
\hline MCAT0.5 & 6.6 & 3.3 & 2.5 & 2 & 2 & 18 \\
\hline MCAT1 & 5 & 5 & 2.5 & 2 & 2 & 18 \\
\hline MCAT2 & 3.3 & 6.6 & 2.5 & 2 & 2 & 18 \\
\hline MCAT10 & 1 & 10 & 2.5 & 2 & 2 & 18 \\
\hline
\end{tabular}

$x$ in MCAT $x$ is molar ratio of Al and Ti in sample.

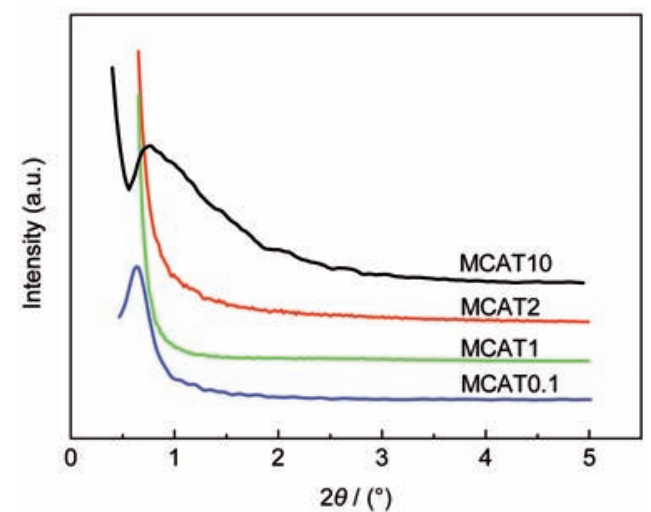

图 1 介孔 $\mathrm{C}-\mathrm{Al}_{2} \mathrm{O}_{3}-\mathrm{TiO}_{2}$ 纳米复合材料的小角 XRD 图

Fig.1 Small-angle XRD patterns of mesoporous $\mathrm{C}-\mathrm{Al}_{2} \mathrm{O}_{3}-\mathrm{TiO}_{2}$ nanocomposites

高的热稳定性, 惰性气氛下高温炦烧去除模板剂可 获得开放的孔道结构.

$\mathrm{C}-\mathrm{Al}_{2} \mathrm{O}_{3}-\mathrm{TiO}_{2}$ 复合材料的小角 XRD 图见图 1. 由 图可知, 复合材料 MCAT2 和 MCAT1 在小角度范围 未见明显的介孔有序结构特征峰, 表明材料的长程 有序程度不高. 材料 MCAT10 和 MCAT0.1 则呈现介 孔有序结构的特征峰, 表明该材料具有较好的有序 度. 而在该多组分体系中, 异丙醇铝和钛酸四丁酯的 水解速度不能同步, 与表面活性剂的分子作用力也 有所不同, 两者添加量相差不大时, 即使通过溶剂挥 发诱导自组装也不能使复合材料变得介观有序. 当 其中某种组分占主要时, 水解速率不同所带来的差 异并不明显, 所以材料仍会显示介观有序的特性. 图 2 为复合材料的大角 XRD 图, 由图可知, 铝含量占主 要的材料 MCAT10 中, 主要由无定形态的碳和氧化 铝组成; 随着钛原子比例的增加, 复合材料呈现一 定的锐钛矿晶型, 从 MCAT0.1 图上可以看到锐钛矿 的(101)、(004)、(200)、(105)和(204)晶面衍射峰.

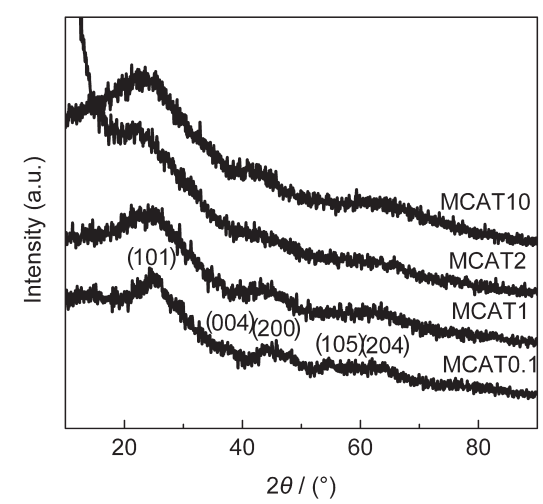

图 2 介孔 $\mathrm{C}_{-}-\mathrm{Al}_{2} \mathrm{O}_{3}-\mathrm{TiO}_{2}$ 纳米复合材料的大角 XRD 图

Fig.2 Wide-angle XRD patterns of mesoporous $\mathrm{C}-\mathrm{Al}_{2} \mathrm{O}_{3}-\mathrm{TiO}_{2}$ nanocomposites 

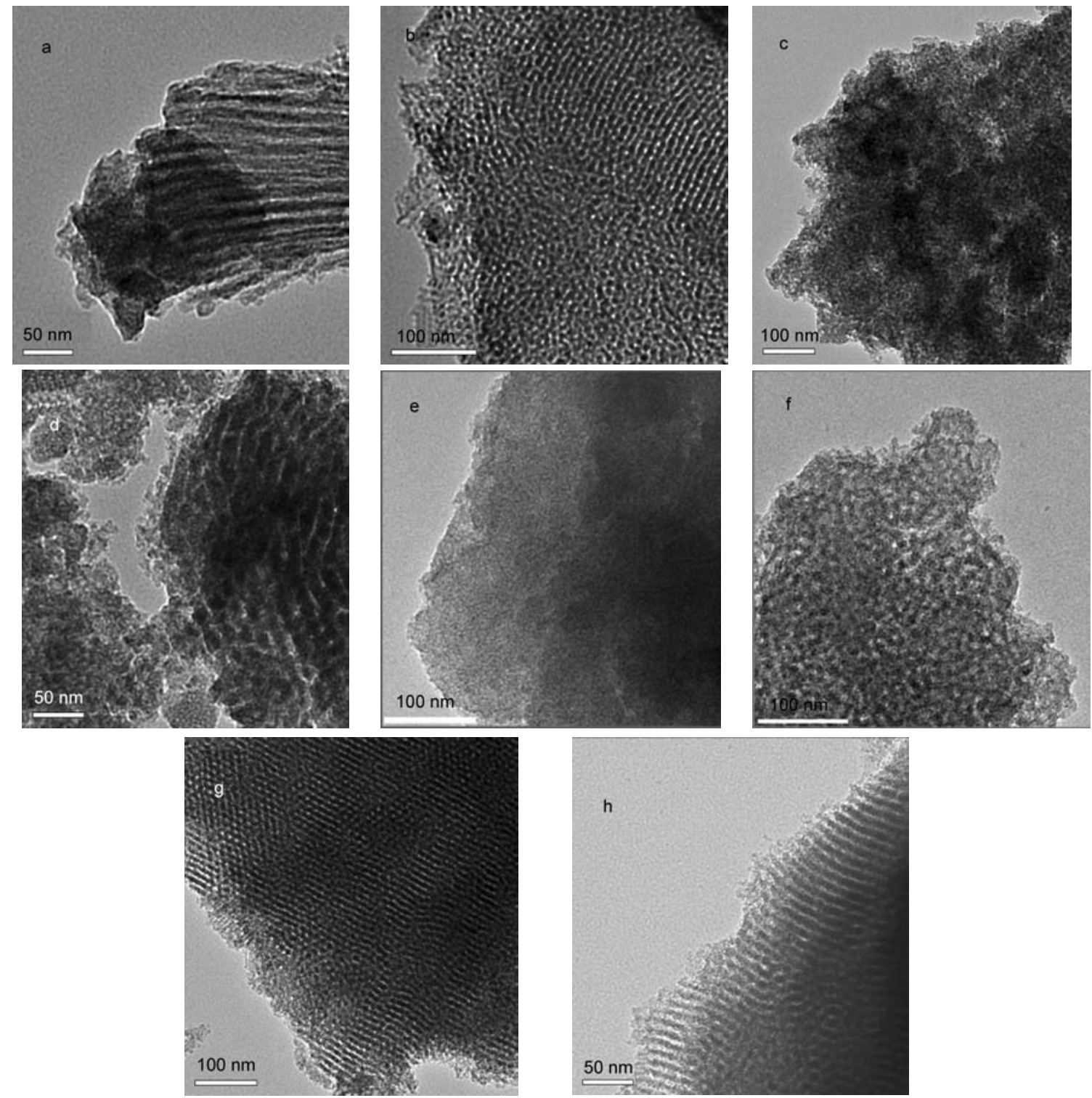

图3 介孔 $\mathrm{C}-\mathrm{Al}_{2} \mathrm{O}_{3}-\mathrm{TiO}_{2}$ 纳米复合材料的 $\mathrm{TEM}$ 图像

Fig.3 TEM images of mesoporpus $\mathrm{C}_{-} \mathrm{Al}_{2} \mathrm{O}_{3}-\mathrm{TiO}_{2}$ nanocomposites

(a, b) MCAT10; (c, d) MCAT1; (e, f) MCAT0.2; (g, h) MCAT0.1

经 $\mathrm{N}_{2}$ 气氛保护下 $500{ }^{\circ} \mathrm{C}$ 热处理的复合材料的 TEM 照片如图 3 所示. 可以从照片上明显地看出

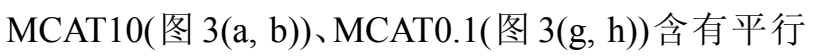
排列的一维孔道(介孔材料的 [001]方向). 当加入 $1<$ $n(\mathrm{Al}): n(\mathrm{Ti})<10$ 时, 由于体系复杂, $\mathrm{Al}$ 前驱体和 $\mathrm{Ti}$ 前 驱体的水解速度不同, 破坏了碳的网络结构, 最后衫 烧得到的复合材料呈现蠕虫状孔道, 与小角 XRD 的 结果一致, 从后面的 $\mathrm{N}_{2}$ 吸脱附等温线也可以发现其 存在结构缺陷. 而当 $\mathrm{Al}$ 前驱体或 $\mathrm{Ti}$ 前驱体相对含量 很低时, 制备的介孔 $\mathrm{C}-\mathrm{Al}_{2} \mathrm{O}_{3}-\mathrm{TiO}_{2}$ 纳米复合材料 MCAT10 和 MCAT0.1 具有一定的有序结构.

图 4 为复合材料 MCAT10、MCAT0.1 的 SEM 图
像. 由图可以看出, 材料呈不规则的块状. 与 MCAT10 相比, MCAT0.1 的表面较为光滑. 图 5 为复 合材料 MCAT10、MCAT0.1 的能量散射光电子能谱 (EDS) 谱图及元素的质量百分比和原子百分比, 可 以看出, $\mathrm{Al}$ 和 $\mathrm{Ti}$ 的实际原子比与理论值大体接近, 但存在一定偏差, 原因是能谱所显示的为材料表面 的原子比, 而由于异丙醇铝与钛酸四丁酯的水解不 同步造成材料表面原子的原子组成与整体不一致.

图 6 和图 7 分别是介孔 $\mathrm{C}-\mathrm{Al}_{2} \mathrm{O}_{3}-\mathrm{TiO}_{2}$ 纳米复合 材料的 $\mathrm{N}_{2}$ 吸附-脱附等温线以及由脱附曲线所得到 的孔径分布图. 可以看出, 所制备样品的 $\mathrm{N}_{2}$ 吸附-脱 附等温线除 MCAT1 以外呈现 Langmuir IV 型吸附 

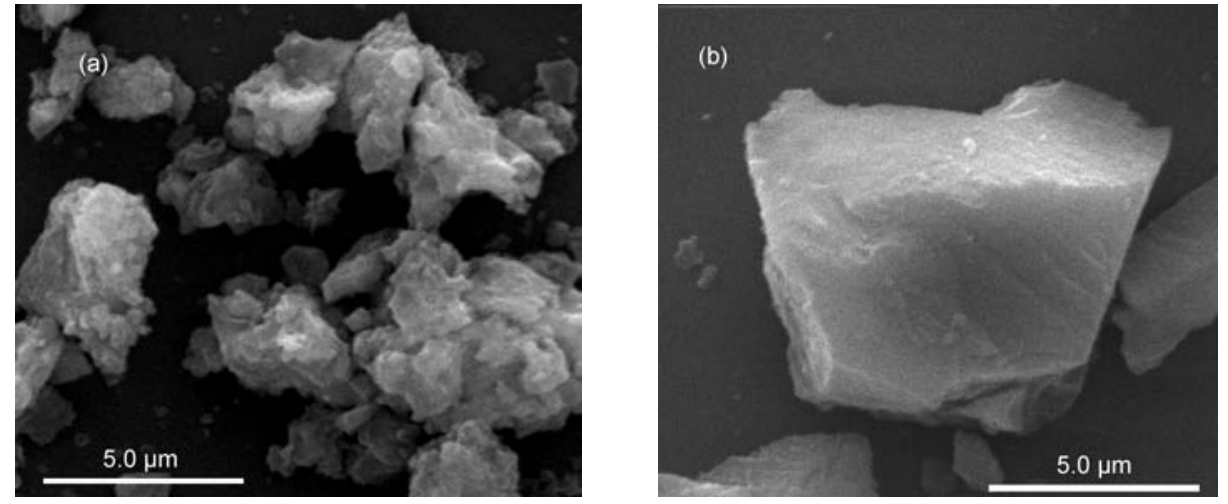

图 4 样品(a) MCAT10 和(b) MCAT0.1 的 SEM 照片

Fig.4 SEM images of (a) MCAT10 and (b) MCAT0.1
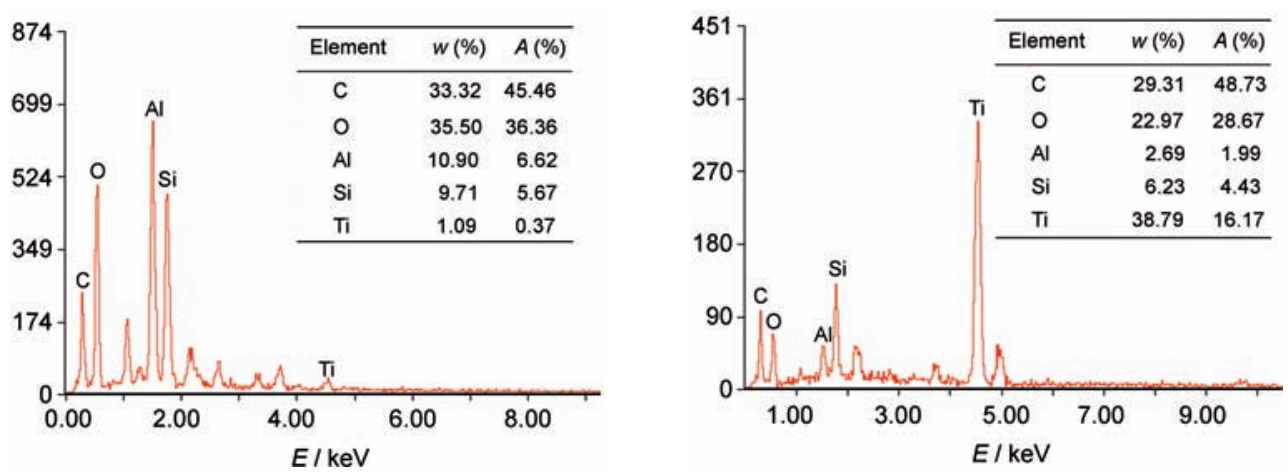

图 5 样品(a) MCAT10 和(b) MCAT0.1 的能量散射光电子能谱图及 $\mathrm{C}, \mathrm{O}, \mathrm{Al}, \mathrm{Si}, \mathrm{Ti}$ 元素的质量分数 (w)与原子分数 $(A)$

Fig.5 Energy dispersive X-ray spectrum (EDS) of (a) MCAT10 and (b) MCAT0.1 and mass fraction (w) and atomic fraction $(A)$ of elements $\mathrm{C}, \mathrm{O}, \mathrm{Al}, \mathrm{Si}$, and $\mathrm{Ti}$

曲线和 $\mathrm{H}_{1}$ 型滞后环, 在相对分压为 0.6-0.8 附近的 $\mathrm{N}_{2}$ 吸附-脱附量有个突跃点, 表明其属于典型的介孔 材料. 非常有趣的是, 从材料的孔径分布曲线(图 5) 可以看出, 制备得到的介孔 $\mathrm{C}-\mathrm{Al}_{2} \mathrm{O}_{3}-\mathrm{TiO}_{2}$ 纳米复合

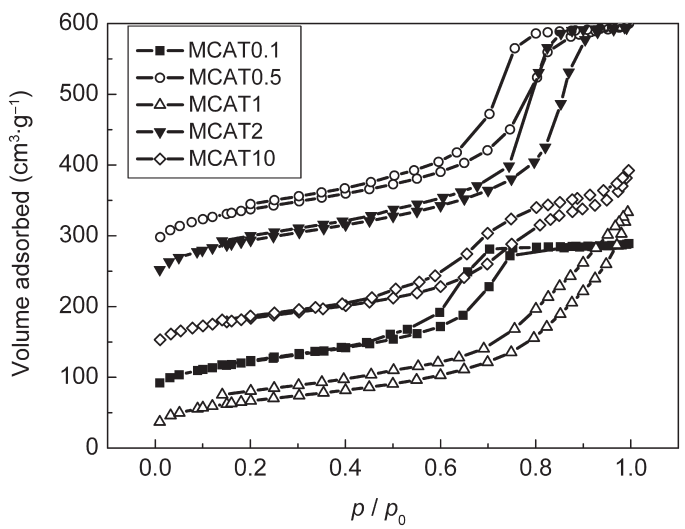

图 6 介孔 $\mathrm{C}-\mathrm{Al}_{2} \mathrm{O}_{3}-\mathrm{TiO}_{2}$ 纳米复合材料的 $\mathrm{N}_{2}$ 吸附-脱附 等温曲线

Fig.6 $\mathrm{N}_{2}$ adsorption/desorption isotherms of mesoporous $\mathrm{C}-\mathrm{Al}_{2} \mathrm{O}_{3}-\mathrm{TiO}_{2}$ nanocomposites

The isotherms for the MCAT0.1, MCAT0.5, MCAT1 and MCAT2 are offset vertically by $50,150,200$, and $100 \mathrm{~cm}^{3} \cdot \mathrm{g}^{-1}$, respectively.
材料有 2 个比较集中的孔径分布. MCAT $1 \mathrm{~N}_{2}$ 吸脱附 等温线的滞后环呈现 $\mathrm{H}_{3}$ 型特征, 表明材料孔道不均 匀, 存在介孔结构的坍塌或堵塞 ${ }^{[24]}$, 与 TEM 测试的

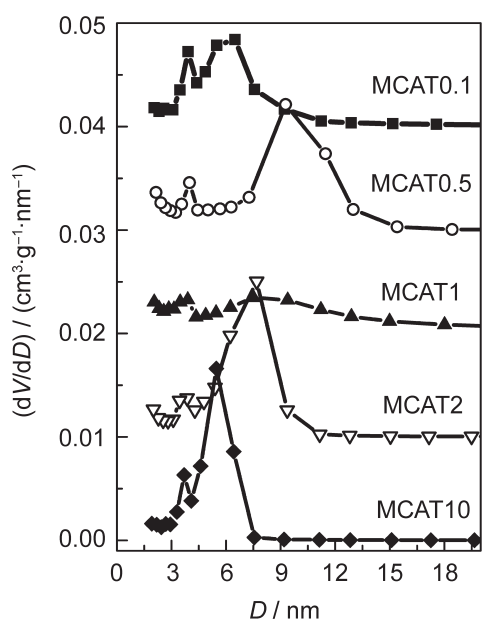

图 7 介孔 $\mathrm{C}-\mathrm{Al}_{2} \mathrm{O}_{3}-\mathrm{TiO}_{2}$ 纳米复合材料的孔径分布

Fig.7 Pore size distribution of mesoporous $\mathrm{C}_{-}-\mathrm{Al}_{2} \mathrm{O}_{3}-\mathrm{TiO}_{2}$ nanocomposites

The isotherms for the MCAT0.1, MCAT0.5, MCAT1 and MCAT2 are offset vertically by $0.4,0.3,0.2$, and $0.1 \mathrm{~cm}^{3} \cdot \mathrm{g}^{-1} \cdot \mathrm{nm}^{-1}$, respectively. 
结果一致.

表 2 总结了介孔 $\mathrm{C}-\mathrm{Al}_{2} \mathrm{O}_{3}-\mathrm{TiO}_{2}$ 纳米复合材料由 氮气脱附曲线计算所得的孔道结构参数, 包括比表 面积、孔容、双孔径分布和介孔面积. 其中 MCAT2 的 BET 比表面积最大达到 $333 \mathrm{~m}^{2} \cdot \mathrm{g}^{-1}$, 其总的孔容 为 $0.63 \mathrm{~cm}^{3} \cdot \mathrm{g}^{-1}$, 介孔的比率由介孔孔容占总孔容的 分数计算得出, 达到 $91.3 \%$, 其孔径主要分布在 3.8 和 $7.7 \mathrm{~nm}$. 比较这组样品可以看出, 当复合材料中氧 化钛占绝对多或氧化铝占绝对多时, 样品的孔径分 布较集中, 且从 TEM 图可以看到孔道较好; 当复合 材料氧化钛和氧化铝含量差不多时, 此时两个孔径 分布相离比较远, 可能由于体系复杂, 异丙醇铝的水 解和钛酸四丁酯的水解分别进行且速度不一样, 具 体机理有待探讨.

\section{2 介孔 $\mathrm{C}-\mathrm{Al}_{2} \mathrm{O}_{3}-\mathrm{TiO}_{2}$ 纳米复合材料的红外发射率}

本文将具有较低发射率的氧化铝、氧化钛引入 介孔碳中. 采用在制备介孔碳的过程中加入金属醇 盐的方法, 制备了介孔 $\mathrm{C}-\mathrm{Al}_{2} \mathrm{O}_{3}-\mathrm{TiO}_{2}$ 纳米复合材料, 从而利用介孔碳中含有红外发射率较低的金属氧化 物来降低介孔材料的红外发射率.

红外隐身涂料中粘结剂的红外透过率越大, 其 红外发射率越小. 故选择红外透过率高的三元乙丙 橡胶 (EPDM) 作防红外涂料成膜物 质 ${ }^{[25]}$, 将介孔 $\mathrm{C}-\mathrm{Al}_{2} \mathrm{O}_{3}-\mathrm{TiO}_{2}$ 纳米复合材料与 $\mathrm{EPDM}$ 混匀, 以该介孔 复合材料为填料, 与粘结剂混合制备涂层, 研究了干 膜厚度为 30 和 $40 \mu \mathrm{m}$ 的涂层在 8-14 $\mu \mathrm{m}$ 波段的平 均红外发射率. 结果如图 8 所示. 材料的红外发射率 随着涂层厚度的增加略有增加. 与介孔碳 $\mathrm{OMC}$ 相 比, 添加金属氧化物的 MCA 和 MCT 的红外发射率 降低, 干膜厚度为 $30 \mu \mathrm{m}$ 时, 红外发射率从 0.625 分 别降低到 0.602 和 0.511 . 氧化铝和氧化钛纳米粒子 在介孔碳中的分散, 可以增大涂层对红外光的散射, 从而有利于降低复合材料的红外发射率. 介孔

表 2 介孔 $\mathrm{C}-\mathrm{Al}_{2} \mathrm{O}_{3}-\mathrm{TiO}_{2}$ 纳米复合材料孔结构参数

Table 2 Pore structure parameters of mesoporpus $\mathrm{C}-\mathrm{Al}_{2} \mathrm{O}_{3}-\mathrm{TiO}_{2}$ nanocomposites

\begin{tabular}{lccccccc}
\hline Sample & \multicolumn{2}{c}{$D / \mathrm{nm}$} & $\frac{S_{\text {BET }}}{\left(\mathrm{m}^{2} \cdot \mathrm{g}^{-1}\right)}$ & & $\frac{S_{\text {meso }}}{\left(\mathrm{m}^{2} \cdot \mathrm{g}^{-1}\right)}$ & $R_{\text {meso }}(\%)$ & $V /\left(\mathrm{cm}^{3} \cdot \mathrm{g}^{-1}\right)$ \\
\hline MCAT0.1 & 3.9 & 6.5 & 259 & 246 & 95.0 & 0.37 \\
MCAT0.5 & 4.0 & 9.3 & 314 & 301 & 95.9 & 0.54 \\
MCAT1 & 3.9 & 7.5 & 236 & 209 & 88.6 & 0.52 \\
MCAT2 & 3.8 & 7.7 & 333 & 304 & 91.3 & 0.63 \\
MCAT10 & 3.7 & 5.5 & 292 & 248 & 84.9 & 0.45 \\
\hline
\end{tabular}

$D$ : pore diameter, $S_{\text {вEт: }}$ BET surface area, $S_{\text {meso }}$ mesoporous area, $R_{\text {meso }}$ : mesoporous percentage, $V$ : total pore volume

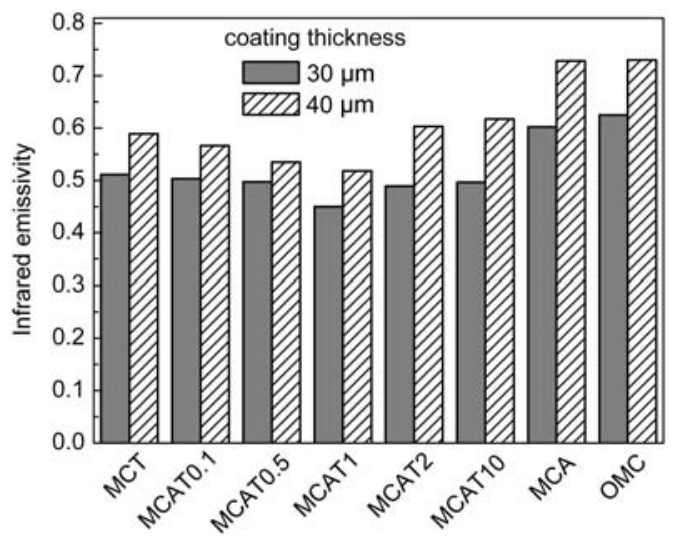

图 8 EPDM/MCAT 涂层的红外发射率

Fig.8 The average infrared emissivity of the coatings using EPDM/MCAT

EPDM: ethylene-propylene-diene monomer

$\mathrm{C}-\mathrm{Al}_{2} \mathrm{O}_{3}-\mathrm{TiO}_{2}$ 纳米复合材料的红外发射率相对于单 一的碳-金属氧化物纳米复合材料进一步降低, 铝和 钛原子比例越接近, 材料的红外发射率越低, 并且低 于单一金属氧化物存在时的发射率. 当铝钛原子摩 尔比为 $1: 1$ 时,涂层的红外发射率最低. 由其 TEM 图 像可以发现, 样品 MCAT1 的孔道结构并不好, 甚至 存在孔道的坞塌和堵塞, 但其在干膜厚度为 $30 \mu \mathrm{m}$ 时, 红外发射率最低为 0.450 . 究其原因, 主要是由于 材料的红外发射率是材料表面特性的函数 ${ }^{[26]}$, 在 MCAT1 材料的表面, 铝和钛相对含量接近, 二者相 对均匀的存在, 这能充分发挥各自的优势, 从而有效 地降低了材料的红外发射率; 另外由于氧化物纳米 粒子与碳之间以及氧化物与氧化物之间存在的相互 作用, 改变了界面的状态, 相当于形成了新的物质 层, 因此界面层具有与本体不同的红外发射率. 同 时, 纳米粒子具有大的比表面, 界面层的比例大, 因 此整个复合物的红外发射率发生了改变 ${ }^{[18]}$, 铝钛的 摩尔比接近于 1 时, 氧化铝和氧化钛的充分混合形 成更多的界面层, 从而使材料的红外发射率有所降 低. C- $\mathrm{Al}_{2} \mathrm{O}_{3}-\mathrm{TiO}_{2}$ 纳米复合材料与 $\mathrm{EPDM}$ 制备的大部 分涂层红外发射率在 0.450-0.617之间, 预示该纳米 复合材料具备了较好的红外隐身能力.

\section{3 结 论}

结合金属氧化物材料的优异性能和聚合物树脂 的骨架稳定性, 提出在合成介孔双金属氧化物的体 系中添加可溶性的酚醛树脂, 通过溶胶-凝胶共组装 形成了 $\mathrm{C}-\mathrm{Al}_{2} \mathrm{O}_{3}-\mathrm{TiO}_{2}$ 的有序介孔网络结构, 调整铝 源和钛源的摩尔比, 可以得到不同组成的具有双孔 
径分布的介孔 $\mathrm{C}-\mathrm{Al}_{2} \mathrm{O}_{3}-\mathrm{TiO}_{2}$ 纳米复合材料. 其中 MCAT0.1 的结构有序度最好, 其比表面积为 259 $\mathrm{m}^{2} \cdot \mathrm{g}^{-1}$, 孔容为 $0.37 \mathrm{~cm}^{3} \cdot \mathrm{g}^{-1}$, 介孔比例高达 $95.0 \%$, 孔径集中分布在 3.9 和 $6.5 \mathrm{~nm}$. 复合材料中的有机碳 能够有效稳定介孔骨架, 使得材料在高温焙烧后依 然能保持较好的有序介孔结构, 而金属氧化物的存 在使得复合材料的整体红外发射率较低. 将该材料 与 EPDM复合制备的涂层红外发射率集中在 0.450-0.617之间, 预示着其具有较好的红外隐身能 力. 该方法为简便合成具有功能性应用的碳-多元金 属氧化物纳米复合材料提供了新思路.

\section{References}

1 Vinu, A.; Miyahara, M.; Sivamurugan, V.; Mori, T.; Ariga, K. J. Mater. Chem., 2005, 15: 5122

2 Feng, X.; Fryxell, G. E.;Wang, L. Q.; Kim, A. Y.; Liu, J.; Kemner, K. M. Science, 1997, 276: 923

3 Roussel, T.; Pellenq, R. J. M.; Bienfait, M.; Vix-Guterl, C.; Gadiou, R.; Beguin, F.; Johnson, M. Langmuir, 2006, 22: 4614

4 Lu, A. H.; Schmidt, W.; Matoussevitch, N.; Bonnemann, H.; Spliethoff, B.; Tesche, B.; Bill, E.; Kiefer, W.; Schuth, F. Angew. Chem. Int. Edit., 2004, 43: 4303

5 Liu, R. L.; Ren, Y. J.; Shi, Y. F.; Zhang, F.; Zhang, L. J.; Tu, B.; Zhao, D. Y. Chem. Mater, 2008, 20: 1140

6 Kresge, C. T.; Leonowicez, M. E.; Roth, W. J.; Vartuli, J. C.; Beck, J. S. Nature, 1992, 359: 710

7 Wang, J. C.; Xiang, C. S.; Liu, Q.; Pan, Y. B.; Guo, J. K. $A d v$. Funct. Mater., 2008, 18: 2995

8 Liu, Q. L.; Zhang, D.; Fan, T. X. Appl. Phys. Lett., 2008, 93 : 013110

9 Zhou, J. H.; He, J. P.; Li, G. X.; Wang, T.; Sun, D.; Ding, X. C.; Zhao, J. Q.; Wu, S. C. J. Phys. Chem. C, 2010, 114: 7611

10 Fang, Z. G.; Li, C. S.; Sun, J. Y.; Zhang, H. T.; Zhang, J. S. Carbon, 2007, 45: 2873

11 Yang, J.; Shen, Z. M.; Hao, Z. B. Carbon, 2004, 42: 1882
12 Yu, H. J.; Xu, G. Y.; Shen, X. M.; Yan, X. X.; Chen, C. W. Appl. Surf. Sci., 2009, 255: 6077

13 Wang, Z. R.; Yu, D. B.; Yu, D. H.; Zhu, X. Y.; Ye, X. Y. Infrared Technology, 1999, 2l(1): 49 [王自荣, 余大斌, 於定华, 朱晓颖, 叶熙源. 红外技术, 1999, 2I(1): 41]

14 Liu, R. L.; Shi, Y. F.; Wan, Y.; Meng, Y.; Zhang, F. Q.; Gu, D.; Chen, Z. X.; Tu, B.; Zhao, D. Y. J. Am. Chem. Soc., 2006, 128: 11652

15 Liu, R. L.; Ren, Y. J.; Shi, Y. F.; Zhang, F.; Zhang, L. J.; Tu, B.; Zhao, D. Y. Chem. Mater., 2008, 20: 1140

16 Wang, T.; Zhou, J. H.; Wang, D. J.; Sun, D.; Di, Z. Y.; He, J. P. Acta Phys. -Chim. Sin., 2009, 25: 2155 [王 涛, 周建华, 王道 军, 孙 盾, 狄志勇, 何建平. 物理化学学报, 2009, 25: 2155]

17 Sun, Y. Q.; Zhou, Y. M. Journal of Inorgnic Materials, 2007, 23 (2): 227 [孙艳青, 周铎明. 无机材料学报, 2007, 23(2): 227]

18 Zhu, D. M.; Li, K.; Luo, F.; Zhou, W. C. Applied Surface Science, 2009, 255: 6145

19 Song, X. H.; Yu, D. H. Infrared Technology, 2003, 25(6): 49 [宋兴华, 於定华. 红外技术, 2003, 25(6): 49]

20 Phanga, S. W.; Tadokorob, M.; Watanabeb, J.; Kuramot, N. Synthetic. Met., 2008, 158: 251

21 Phanga, S. W.; Tadokorob, M.; Watanabeb, J.; Kuramot, N. Polym. Adv. Technol., 2009, 6: 550

22 Li, J. M.; Cai, X.; Mao J. F. Materials for Mechanical Engineering, 2004, 28: 34 [李景明, 蔡 殉, 茅及放. 机械工程材料, 2004, 28: 34]

23 Meng, Y.; Gu, D.; Zhang, F. Q.; Shi, Y. F.; Yang, H. F.; Li, Z.; Yu, C. Z.; Tu, B.; Zhao, D. Y. Angew. Chem. Int. Edit., 2005, 44: 7053

24 Rojas, F.; Kornhauser, I.; Felipe, C.; Esparza, J. M.; Cordero, S.; Domingueza, A.; Riccardo, J. L. Phys. Chem. Chem. Phys., 2002, 4: 2346

25 Yu, H. J.; Xu, G. Y.; Shao, C. M.; Chen, C. W.; Hu, C. Infrared Technology, 2008, 30(3): 154 [余慧娟, 徐国跃, 郡春明, 程传 伟, 胡 晨. 红外技术, 2008, 30(3): 154]

26 Dai, G. P. Journal of Sichuan Ordnance, 1996, 17: 2 [戴光平. 四川兵工学报, 1996, 17: 2] 